\title{
Juicy Haptic Design: Vibrotactile Embellishments Can Improve Player Experience in Games
}

\author{
Tanay Singhal \\ University of Waterloo \\ Waterloo, Ontario, Canada \\ tanay.singhal@uwaterloo.ca
}

\author{
Oliver Schneider \\ University of Waterloo \\ Waterloo, Ontario, Canada \\ oliver.schneider@uwaterloo.ca
}

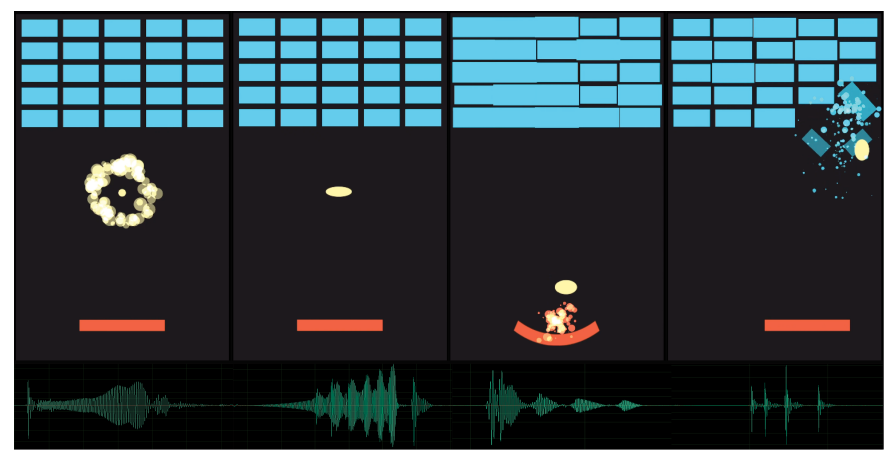

HIGH JUICY VISUALS \& HIGH JUICY HAPTICS

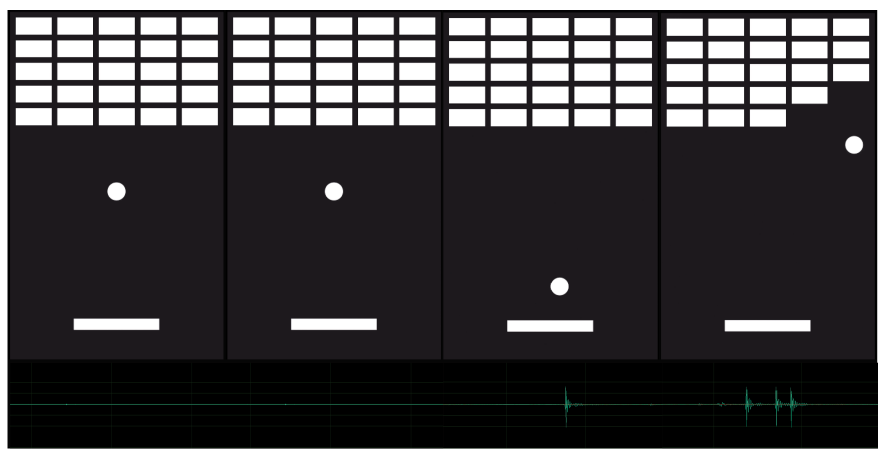

LOW JUICY VISUALS \& LOW JUICY HAPTICS

Figure 1: Two of the six Breakout-style game conditions in study 2; haptic feedback is visualized as waveforms underneath.

\begin{abstract}
Game designers and researchers employ a sophisticated language for producing great player experiences with concepts such as juiciness, which refers to excessive positive feedback. However, much of their discourse excludes the role and value of haptic feedback. In this paper, we adapt terminology from game design to study haptic feedback. Specifically, we define haptic embellishments (HEs) as haptic feedback that reinforce information already provided through other means (e.g., via visual feedback) and juicy haptics as excessive positive haptic feedback with the intention of improving user experience in games and other interactive media. We report two empirical studies of users' experiences interacting with visuohaptic content on their phones to 1) study participants' preferences for ten design principles for HEs and 2) measure the added value of juicy haptics, implemented as HEs, on player experience in a game. Results indicate that juicy haptics can enhance enjoyability, aesthetic appeal, immersion, and meaning.
\end{abstract}

\section{CCS CONCEPTS}

- Human-centered computing $\rightarrow$ Haptic devices; Graphical user interfaces; Empirical studies in HCI.

This is the author's version of the work. It is posted here for your personal use. Not for redistribution. The definitive Version of Record is published by the ACM in the following venue:

\section{KEYWORDS}

haptics; vibrotactile feedback; juiciness; player experience; games ACM Reference Format:

Tanay Singhal and Oliver Schneider. 2021. Juicy Haptic Design: Vibrotactile Embellishments Can Improve Player Experience in Games. In $\mathrm{CHI}$ Conference on Human Factors in Computing Systems (CHI '21), May 813, 2021, Yokohama, Japan. ACM, New York, NY, USA, 11 pages. https: //doi.org/10.1145/3411764.3445463

\section{INTRODUCTION}

Game designers employ a sophisticated language for creating games that have great experiences. Concepts like "game feel" [48], the feeling of immersive real-time control, and "juiciness", a type of game feel that involves abundant audiovisual feedback like screen-shake and particle effects [21,27], can inform the design of new games. Researchers have recently started to study these concepts, finding evidence that juiciness can improve player experience (PX) in some contexts [21,32]. However, despite recent commercial devices like the iPhone, Nintendo Switch, and Sony PS5 featuring high-fidelity haptic actuators, haptic feedback has remained strikingly absent from the conversation.

There is a small, but growing, body of quantifiable evidence that haptic feedback can contribute to other multimedia experiences. With movies, mid-air haptic feedback can make experiences measurably more pleasant, unpredictable, and creative [38], while motion seats can evoke more positive emotions as measured by physiological signals [43]. In virtual reality (VR) environments, passive haptic objects have been linked to increased presence [4], provided it matches visual feedback [7].

There are also encouraging, though informal, signs that haptic feedback improves PX. On a Reddit thread about haptics in iPhone 
games, a top-voted comment states that if you "search "haptic feedback' here on Reddit[,] most of the posts or comments say how cool it is" [49]. Game journalists have also specifically commended haptic feedback [35]. Nevertheless, to our knowledge, no formal research has quantitative measures of the impact of haptic feedback on PX.

We study the design of haptic feedback through the lens of juiciness, a concept that is gaining momentum for its potential to improve PX [21, 22, 29, 32, 33]. Surprisingly, existing discourse on juiciness has almost completely neglected the role of haptic feedback, except for suggestions of it as an area for future development $[3,48]$.

In this paper, we aim to bring haptics to the forefront of research on juiciness, specifically, by defining and measuring the added value of juicy haptics on PX. Because haptic feedback can serve highly diverse interaction goals, we limit our scope to purely decorative haptic feedback - what we call haptic embellishments (HEs). Doing so also directly links our research to recent work on juiciness via visual embellishments [21]. We pursue two research questions:

RQ 1: What principles and concepts are effective for designing appealing HEs?

RQ 2: What is the added value of juicy haptics, implemented as HEs, to PX?

We conducted two remote user studies on participants' phones. In study 1, we recruited 26 participants to compare and rank 52 animations with haptic feedback, organized into ten proposed design principles to create juicy haptic embellishments (see Figure 2 and Figure 3). Results indicate a preference for visual and haptic embellishments created with our proposed design principles. In study 2 , we recruited 38 participants to play 6 variations of a Breakout-style game (all pairs of low juicy/high juicy visuals, and low juicy/high juicy/no haptics; see Figure 1) on their phones. We found that juicy haptics can improve PX, specifically, the constructs of aesthetic appeal, immersion, and meaning (see Figure 6).

Our work builds on the efforts to measure the added value of haptics to UX [38]. The results are encouraging the potential of haptics in games research. In short, we contribute:

(1) The first formal evidence that haptic feedback can improve PX - in particular, we found it can improve aesthetic appeal, immersion, meaning, and enjoyability.

(2) Definitions for juicy haptics and haptic embellishments (HEs).

(3) Ten design principles for HEs, including an empirical evaluation on their impacts on user preference.

\section{DEFINING JUICY HAPTICS AND HAPTIC EMBELLISHMENTS}

fuiciness is a concept in game design that is widely defined as "excessive positive feedback" [13, 28, 32]. Even though there is some disagreement on a specific definition [21,33, 48], many prior works $[19,27,32]$ quote Gray et al's description for what a juicy game should feel like:

A juicy game element will bounce and wiggle and squirt and make a little noise when you touch it. A juicy game feels alive and responds to everything you do - tons of cascading action and response for minimal user input [17].
Although juiciness is usually discussed in the context of game design, we believe it is also a useful concept for designing feedback in other interactive media such as animations or movies. Thus, we define juicy haptics as:

Excessive positive haptic feedback with the intention of improving user experience in games and other interactive media.

Because "haptic feedback" can serve highly diverse interaction goals (e.g., to provide, notify, or guide) and roles (e.g., complementary, reinforcing, initial, primary, secondary) [37], we limit the scope of our study on juicy haptics to purely decorative haptic feedback. We define haptic embellishments (HEs) as:

Haptic feedback that reinforces information that is already provided through other means.

We based this definition on "visual embellishments", which have been studied in designing information charts [5] and juicy games [21]. Examples of HEs include the satisfying haptic feedback when you complete a transaction using Apple Pay or the intense rumbling of a controller during an Earth-shattering explosion effect in a game. Conversely, an example of haptic feedback that is not an embellishment can be found in the 1-2-Switch game, Safe Crack, which requires feeling haptic feedback to unlock a safe. In this case, the haptic feedback provides new and necessary information that is not provided by other means.

\section{RELATED WORK}

Here, we describe related work on haptic feedback in multisensory experiences, haptics in games and other interactive media, and juiciness in games literature.

\subsection{Vibrotactile Feedback in Multisensory Experiences}

Haptics is a multimodal experience, with a tight link between visual, audio, and haptic feedback. Maclean et al. review haptic systems with a focus on multisensory design [37], providing vocabulary and guidance to designers on how to choose the role of haptics in a multisensory system. Berger et al. proposed an "uncanny valley" of haptics, where experience (measured as presence) decreases when visual and haptic feedback do not have the same spatial fidelity [7]. Chang \& Sullivan found that the inclusion of haptics seems to enhance the perception of audio quality [11].

Research into vibrotactile feedback has traditionally focused on utility and usability, rather than experience. From the first characterizations of haptic icons [36] and their vibrotactile analogue, tactons [8], the focus was on information content and organizational principles to represent various abstract meanings and measuring recognition rates [9]. Increasingly, emotion and affect have become guiding principles for haptic design [15]. Much of this work focused on either recognition of the intended emotion [44] or the mapping of design parameters like amplitude and frequency onto an emotional space involving arousal and valence [50]. "Emotion" is one of the four main facets proposed by Seifi \& MacLean, alongside more pragmatic concerns: sensory attributes, metaphoric association, and usage examples [47]. Israr et al. explore the experiential role of haptics through feel effects, a pairing between linguistic phrases and 
haptic patterns [25]. Although feel effects are intended for use in an experience such as storytelling, Israr et al. study them through isolated phrases. Most recently, Kim \& Schneider proposed the Haptic Experience (HX) Model, suggesting five experiential dimensions for a good haptic experience: Harmony, Autotelics, Immersion, Realism, and Expressivity [34].

\subsection{Haptics in Games and Other Interactive Media}

Although no work has studied the added value of haptics to player experience (PX), haptic feedback has improved experience in a number of interactive media such as mobile interfaces [11], virtual reality [4], audiovisual clips [38], and motion seats [43]. In motion seats, Pauna et al. showed that physiological signals of positive emotions increased with motion seat feedback [43]. In movie clips, Maggioni et al. discovered that adding mid-air haptics or vibrotactile feedback made the experience more pleasant, unpredictable, and creative [38]. Maggioni et al.'s work endeavored to rigorously codify the added value of haptic feedback - to do so, they used the AttrakDiff questionnaire [20], which measures an experience's overall attractiveness. However, AttrakDiff is insufficient to measure player experience constructs such as immersion or mastery.

Most existing work on haptics in games has focused on designing hardware or systems rather than conducting studies to measure PX. For example, Park et al. created custom haptic hardware for a "Brickout" (Breakout-style) game [42]; Andrews et al. designed a game where players assume the role of a wizard with a hapticenabled wand [2]. Israr \& Stec designed a chair with a grid of vibrotactile actuators and demonstrated the system in a racing game, using haptic feedback for events such as road bumps, acceleration, collisions, and engine throttling [23]. Israr et al. explored using a physical device to feel motion across hands and vibrotactile gloves to feel illusory sensations of mid-air objects [24]. However, none of the aforementioned works conducted user studies.

Eid et al. [14] designed a multiplayer shooter game with tactile and kinesthetic feedback; they reported that participants preferred using the Falcon device over the keyboard and that a haptic jacket made the experience more realistic, but their study procedure is unclear and their questionnaire consists of only four informal questions. Other research has focused on improving accessibility of games through novel haptic devices [10, 39, 45].

\subsection{Juiciness in Games Literature}

Juiciness is a concept in game design that refers to "excessive positive feedback" $[13,28,32]$, though there is some disagreement on the exact definition. For example, Swink et al. [48] argue that the excessive feedback should be a balance of positive and negative feedback. More recently, Hicks et al. interviewed 17 game developers to define juiciness as a "phenomenon that emerges from coherent design of game mechanics and visuals, while providing confirmatory, explicit and ambient feedback" [21,33], but they note that "some more detailed elements of this definition remain intangible" [21].

Hicks et al. [21] found some evidence that juiciness, implemented as visual embellishments (e.g., particle and animation effects), can improve a game's visual appeal and PX constructs such as curiosity, immersion, and meaning. A preliminary study by Juul \& Begy [29] compared a juicy versus non-juicy version of a tile-matching game with audiovisual feedback - players rated the quality of the juicy version higher (mean 3.74 versus 3.26 out of 5), but the results were not statistically significant. More recently, Kao et al. [32] compared four levels (None, Medium, High, and Extreme) of juiciness, implemented as audiovisual feedback, on an action role-playing game. They found that the None and Extreme conditions significantly decreased PX relative to the Medium and High conditions, suggesting that juiciness should be used in moderation.

Most research on juiciness is limited to audiovisual feedback, ignoring haptic feedback. For example, Hicks et al. describe juiciness as "the provision of abundant audiovisual feedback" [22] or "the idea that large amounts of audiovisual feedback contribute to a positive PX” [33]. In a different paper, Hicks et al. [33] briefly acknowledge haptic feedback as a form of multimodal feedback that can contribute to juiciness, without further elaboration. Other studies on juiciness $[3,29,32]$ also concentrate on audiovisual feedback, although Atanasov [3] discusses haptics in Further work: "there is obviously a ground for exploration of haptic feedback, especially in regard to 'Juiciness'."

\section{THE TEN PRINCIPLES OF HAPTIC EMBELLISHMENTS}

Designing haptic feedback is a challenging task for novice hapticians [46], in part because of the scarcity of practical guidelines on haptic design. To this end, we assembled ten principles for designing HEs in multisensory experiences through iterative brainstorming and a literature review. See Figure 2, Figure 3, and the Video Figure (includes audible haptic feedback) for example implementations.

We draw heavily from past work on visual and haptic design, including: Disney's twelve basic principles of visual animation [26, Chapter 3], the principles of haptic cinematography [18], Apple's WWDC 2019 talk on designing audio-haptic experience [40], and the experiential dimensions of haptic experience [34]. For some background, Disney's twelve basic principles of animation [26, Chapter 3] were immensely influential to the 3D graphics and animations community: the principles are highly cited in SIGGRAPH literature and widely taught to animation students.

Similar to Disney's principles, our principles give hapticians a conceptual framework for designing haptic embellishments in games, animations, and other multisensory experiences. The principles are neither exhaustive nor prescriptive, and need not be used all at once - one must still iterate and exercise judgment. Finally, though we study them as vibrotactile feedback, their ideas should apply to other haptic modalities.

Realism. Haptic parameters such as intensity, frequency, temperature should be used to describe an object's physical qualities. For example, low frequency vibrations communicate softness or weakness while high frequency vibrations indicate density and strength [41]. In that case, an animation of a squishy ball bouncing should be paired with low frequency vibrations and a marble ball with high frequency feedback.

Anticipation. Haptics can be used to build anticipation and excitement for an upcoming action. For example, in the Apple Watch alarm, a ramp-up vibration builds anticipation for the upcoming 
audio feedback [40]. In movies and animations, an example of anticipation is a baseball player character needing to swing their bat backwards before striking the ball or the eerie music in a horror movie before a jump scare.

Staging. Design haptic feedback to draw a user's attention to a particular object, character, or action. In visuals, this can be achieved by zooming into an object or priming it with particle effects. Similarly, haptic feedback can be used to drag the user's attention towards a scene in animations [18] prior to an action.

Follow-Through. Use haptic feedback to communicate an animation's after-effects. Because of inertia, objects tend to keep moving even after completing their main motion. For example, after releasing a slingshot, the elastic strips continues to vibrate, which can be emphasized through an oscillating vibration effect.

Slow In and Out. Objects in the real-world, such as vehicles and human bodies, rarely move at constant speed because they need time to accelerate and slow down. This principle is widely used to make smoother and more organic visual animations. Similarly, we hypothesize that vibrotactile feedback can also benefit from this principle such as in the trajectory of vibration intensity. (Guillotel et al. include slow in and out under Kinetics [18]).

Exaggeration. Use haptic feedback to present objects and actions in a wilder, more extreme form. Take the example of an animation of a walking giant, crushing all vehicles and buildings underneath its mighty feet. Haptic feedback can communicate the giant's ferocity by including very intense rumbles with its enormous footsteps.

Synchronism. When intending to play haptic feedback at the same time as other feedback, one must synchronize it as precisely as possible. It is quite unpleasant to watch a video with misaligned audio, even if only by a fraction of a second. The same is true for haptic feedback $[11,18]$ - our results for study 1 show that even a 0.15 second error in synchronism is disconcerting.

Timing. That being said, haptic feedback does not always have to play at the same time as other feedback. Experiment with different timings to build anticipation before an event or communicate followthrough effects afterwards. Moussette \& Verweij describe haptics and audio as instruments that do not "always have to play the same thing, but they do have to play at the same tempo" [40].

Energy. Haptic feedback should match the energy level that an object should convey. An attention-seeking visually pulsating object pairs well with a faster-paced, more intense vibrations, and high frequency vibration.

Expressivity. Haptic feedback should distinctly reflect varying user input and system events [34]. In an animation with a small pebble and large boulder falling, the associated haptic feedback should feel clearly contrasted. Beyond differences in intensity and frequency when the rocks land, also consider variations in timing and timbre [34]. Which one attracts more attention and energy as it falls? How will the two rocks bounce? Which one will cause more damage? How will their aftershocks feel, if any?

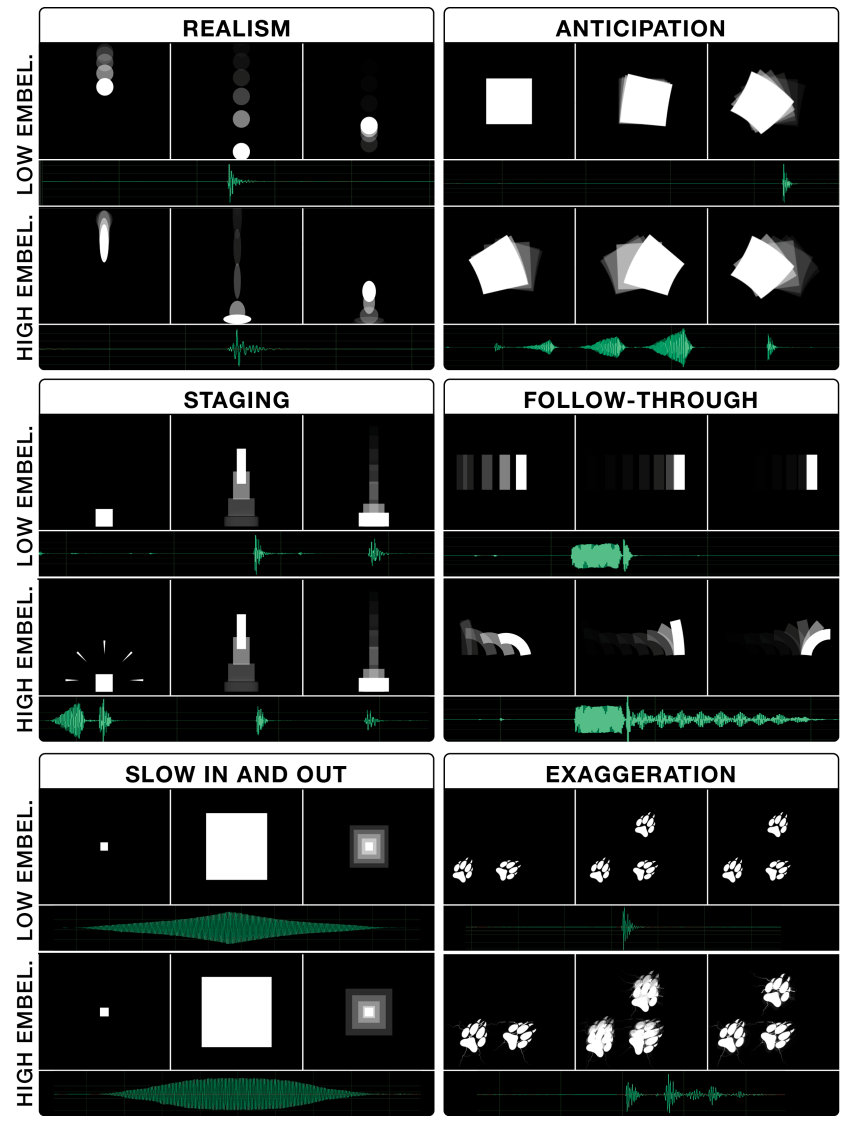

Figure 2: Implementations from the six visuo-haptic design principles from in study 1 . Under each principle are low and high embellishment visuals (three animation frames) and haptics (green waveform underneath). See the Video Figure for the full animations with audible haptic feedback and Supplemental Material for all source files.

\section{STUDY 1: EVALUATING THE DESIGN PRINCIPLES OF HAPTIC EMBELLISHMENTS}

To understand how to design haptic embellishments for juicy haptics, we first answer RQ 1: What principles and concepts are effective for designing appealing HEs?

We conducted a study to understand user preferences for implementations of each of our ten design principles, including possible multimodal confounds and interaction effects between visual and haptic embellishments.

\subsection{Participants}

We recruited 26 participants through social media websites (Facebook and Reddit) as well as our own personal and professional network. The eligibility criteria required individuals between the age of 18 to 64 with an iPhone 8 or newer, for which we designed 

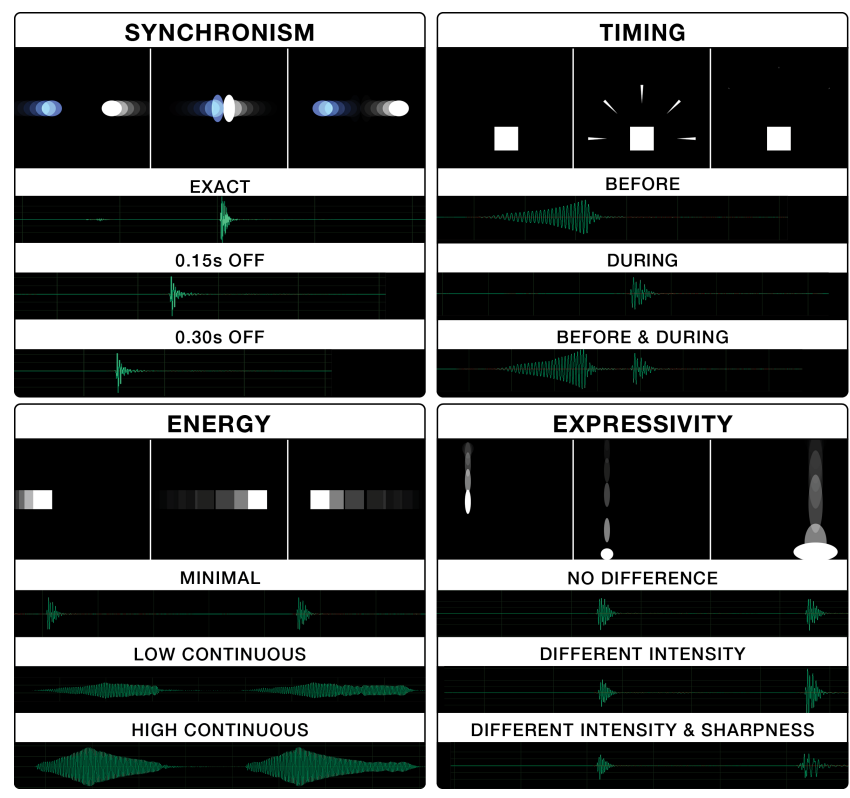

Figure 3: Implementations of all four haptic-only design principles in study 1 . Under each principle is one visual animation with three different haptic embellishments. See the Video Figure for the full animations with audible haptic feedback and Supplemental Material for all source files.

the stimuli and study (see Materials for a rationale). Of the participants, 15 self-identified as women and 11 as men. Participants were 18 to 34 years old with limited familiarity with haptics.

\subsection{Materials}

Device. We designed the stimuli for the Apple iPhone 8 or newer, which comes with the Taptic Engine-a custom linear resonant actuator (LRA) that produces small vibrations by moving a mass back and forth along a single axis. Apple's Core Haptics framework enables developers to create transient (short, one-time vibration) and continuous (with customizable duration) vibrotactile effects that can be finely controlled across both intensity and sharpness (i.e., the frequency) over time using parameter curves.

Stimuli. We designed 52 visuo-haptic animation clips in total. We created the visual animations using After Effects and haptic embellishments by writing object notation in the Apple Haptic and Audio Pattern (AHAP) file format, which can be rendered using the Core Haptics framework. See Supplemental Materials for the source files.

Visuo-haptic principles. We refer to six of our ten principles (Realism, Anticipation, Staging, Follow-Through, Slow In and Out, and Exaggeration) as visuo-haptic principles because they apply to both visuals and haptic. For each of these principles, we designed six visuo-haptic animation clips for all combinations of three haptic embellishment conditions (none, low embellishment, high embellishment) and two visual embellishment condition (low embellishment, high embellishment). See Figure 2 and the Video Figure to view all the visuo-haptic stimuli.

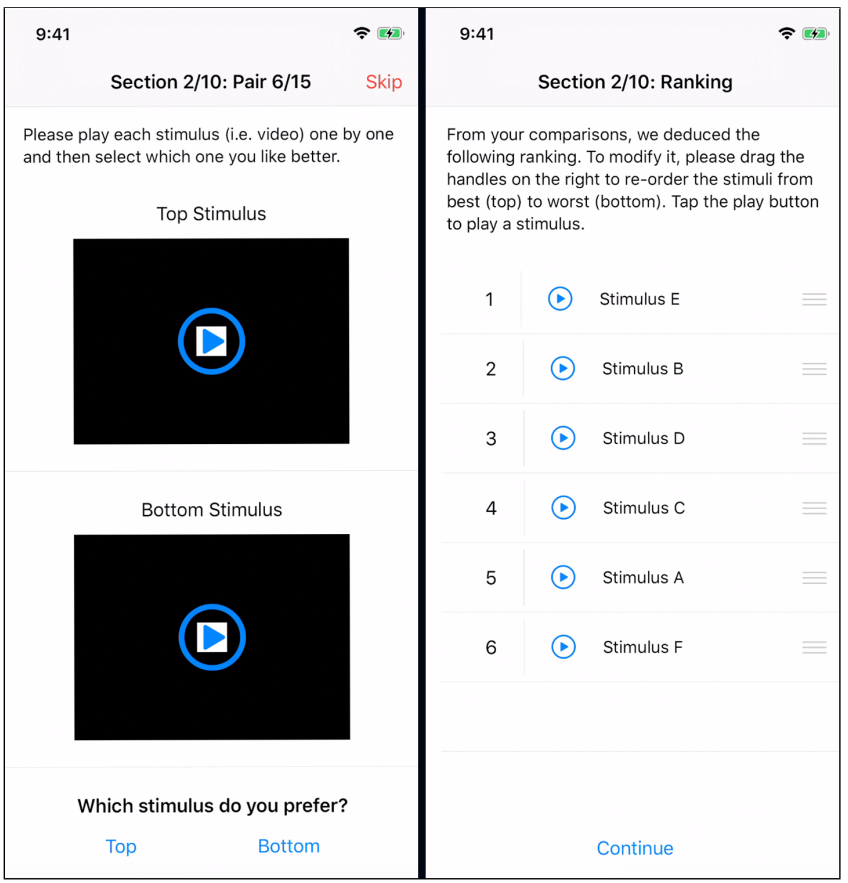

Figure 4: Screenshots of the app we created to deliver study 1. Left: participants compare two stimuli and pick their favourite; right: participants can re-play and rank stimuli after completing comparisons for a principle.

Haptic-only principles. We refer to four of our ten principles (Synchronism, Timing, Energy, and Expressivity) as haptic-only principles because they refer to design decisions for haptic embellishments only while keeping the visuals constant. For each of these principles, we designed four visuo-haptic animation clips that vary across four different implementations of haptic embellishment, one of which is "no haptics". See Figure 3 and the Video Figure to view all the haptic-only principles stimuli.

Study App. We created an iOS app using XCode and Swift through which participants can complete the entire study remotely. The app collects consent, checks if vibrations are supported, presents the visuo-haptic animation clips in randomized order, asks our questions (see Procedure), and transfers data to our database. See Figure 4 and the Video Figure for a walkthrough of the interface.

\subsection{Procedure}

We send participants our custom-built iOS app (see Materials and the Video Figure) for completing the entire study remotely. At first, the app collects participants' consent and basic demographics questions (age, gender, and professional/academic experience in haptics). Next, the app automatically checks the device's compatibility with the Core Haptics framework. It then prompts participants to try a test vibration and verify that they felt it (in case haptics is disabled in their device's settings). If the device is incompatible or the participants reports that they could not feel the test vibration, participants are disqualified from the study. 
After this, the app presents the study into two stages. In stage 1 (shown as sections 1-6 in the Video Figure), participants are shown stimuli (i.e., visuo-haptic animation clips) for visuo-haptic principles, while in stage 2 (shown as sections 7-10 in the Video Figure), participants are presented stimuli for haptic-only principles. Within each stage, the app presents the principles one-by-one in randomized order. Within each principle, participants are shown all possible pairs of the different stimuli $\left(\left(\begin{array}{l}6 \\ 2\end{array}\right)=15\right.$ pairs for visuo-haptic principles and $\left(\begin{array}{l}4 \\ 2\end{array}\right)=6$ pairs for haptic-only principles) and asked to pick their preferred stimuli (see Figure 4). Both the order of the pairs and the order of stimuli within a pair (A/B versus B/A) are randomized. Participants may re-play the pairs of stimuli as often as they wish until they enter a preference or press "SKIP" if they cannot decide. The rationale for asking participants to compare pairs of stimuli first is to reduce the cognitive load of experiencing too many stimuli at once. Once participants have indicated their preferences for all pairs of stimuli within a principle, the app uses their preferences to generate a ranking of the stimuli using the round-robin row-sum scores procedure [12]. At this stage, participants see the rankings - they may replay and re-order the stimuli if they wish (see Figure 4). After rankings are submitted, we ask participants to rate the distinguishability of the stimuli they just experienced ("I was easily able to tell apart all the stimuli that I just compared and ranked") on a 5-point Likert scale. This process repeats for each of the principles.

\subsection{Results}

5.4.1 Data analysis procedure. No data cleaning was performed. Because the data did not satisfy the normality and homoscedastic assumptions for ANOVA, we used Friedman tests for significance testing, followed by an exact all-pairs test for Friedman tests [16]. Pvalues were adjusted using the Benjamini-Hochberg [6] procedure to reduce the false discovery rate (FDR). For each principle, our data analysis shows that the effect of conditions on rankings are significant $(p<0.001)$.

5.4.2 Visuo-haptic principles. According to Figure 5c, high embellishment visuals and haptic condition (V2H2) had the highestranking mean user preference on average, with statistical significance. We can also see that the two poorest performing conditions were the ones with no haptic feedback. Figure 5a provides insight on each of the six visuo-haptic principles. V2H2 ranked the highest in four of the principles: anticipation (best: V2H2 $\mu=2.04$; secondbest: $\mathrm{V} 1 \mathrm{H} 1 \mu=2.57$ ), staging (best: $\mathrm{V} 2 \mathrm{H} 2 \mu=2.04$; second-best: V2H1 $\mu=2.46$ ), follow-through (best: V2H2 $\mu=2.31$; second-best V2H1: 2.50), and exaggeration (best: V2H2 $\mu=2.23$; second-best V1H1 $\mu=2.62$ ). From the second-bests listed previously, we can see that $\mathrm{V} 2 \mathrm{H} 1$ and $\mathrm{V} 1 \mathrm{H} 1$ were often the second-best conditions.

Distinguishability scores for stimuli within a principle, ordered from least distinguishable to most distinguishable, are as follows: slow in and out $(\mu=2.65)$, realism $(\mu=3.46)$, anticipation $(\mu=3.81)$, staging $(\mu=4.00)$, exaggeration $(\mu=4.04)$, and follow-through $(\mu=4.08)$. In other words, participants struggled most with distinguishing stimuli within slow in and out and realism. Incidentally, slow in and out and realism also produced the most mixed results.
5.4.3 Haptic-only principles. See Figure $5 \mathrm{~b}$ for results on the four haptic-only principles. Synchronism: participants ranked the "exact" synchronism condition as the highest $(\mu=1.50)$. Interestingly, average rankings for unsynchronized haptic feedback is not significantly different from the no haptic condition. Timing: "during" $(\mu=1.88)$ and "before $\&$ during" ( $\mu=1.92)$ were most preferred on average. Energy: "low continuous" ( $\mu=2.04)$ was most preferred, though only by a narrow margin compared to other haptic implementations. Expressivity: "different intensity and sharpness" $(m u=1.81)$ ranked the highest, followed by "no difference" ( $m u=2.19)$.

Distinguishability scores for stimuli within a principle, ordered from least distinguishable to most distinguishable, are as follows: expressivity $(\mu=3.615)$, timing $(\mu=3.85)$, synchronism $(\mu=3.96)$, and energy $\mu=4.04$ ). In other words, participants were mostly able to distinguish the stimuli within the principles (which is a striking result on its own, given that only the haptic feedback changed across stimuli).

\subsection{Key takeaways}

High haptic embellishment (versus low haptic embellishment) enhanced visual stimuli in four of the six visuo-haptic principles: anticipation, staging, follow-through, exaggeration (see Figure 5a). In the four haptic-only principles (see Figure 5b), 1) haptic embellishments and visual embellishments should be synchronized exactly, 2) haptic feedback should be used to communicate an object's energy levels (e.g., motion), and 3) vibrotactile embellishments should vary across both intensity and sharpness for different objects and events (i.e., expressivity).

\section{STUDY 2: JUICY HAPTICS AND PLAYER EXPERIENCE}

In study 1, we evaluated the design principles of HEs individually and gained specific design insight. In study 2, we combined the insights from all ten principles of HEs to implement juiciness and pursue RQ 2: What is the added value of juicy haptics, implemented as HEs, to PX?. To measure added-value, we conducted a within-subjects user study: 38 participants played six versions of a Breakout game-one for each combination of haptic juice (none, low, high) $\times$ visual juice (low, high) - in randomized order and filled out questionnaires on player experience, haptic experience, and enjoyability after each game.

\subsection{Materials}

Device. Same as study 1.

The Six Breakout-style Games. We designed six versions of a Breakoutstyle game: one for each combination of haptic juice (none, low, high) $\times$ visual juice (low, high). The mechanics and controls are identical across games; only visual and haptic embellishments change. We picked a Breakout-style game because 1) it is familiar and easy to play and 2) it builds upon prior work that used a Breakout-style game to describe audiovisual juice effects [27]. In the game, the player drags a paddle with their finger, with the intention of aiming the ball at bricks, which destroy on contact with the ball. The game does not have win or lose conditions - it restarts if all the bricks are 


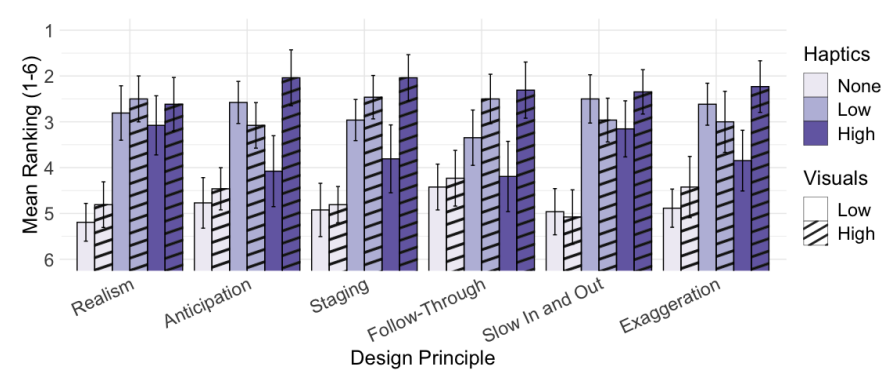

(a) Mean rankings for the six design principles that vary across visual \& haptic embellishment levels (i.e., visuo-haptic principles).

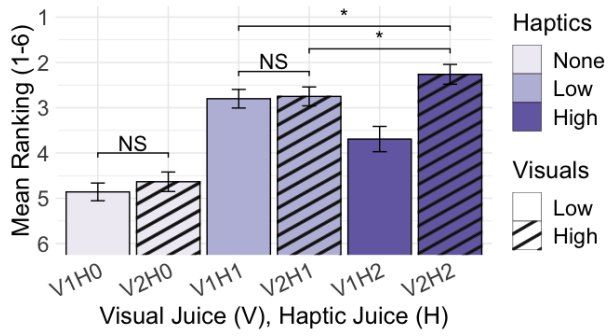

(c) Mean rankings of the six visuo-haptic principles (aggregated) across visual \& haptic embellishment levels. NS = not significant.

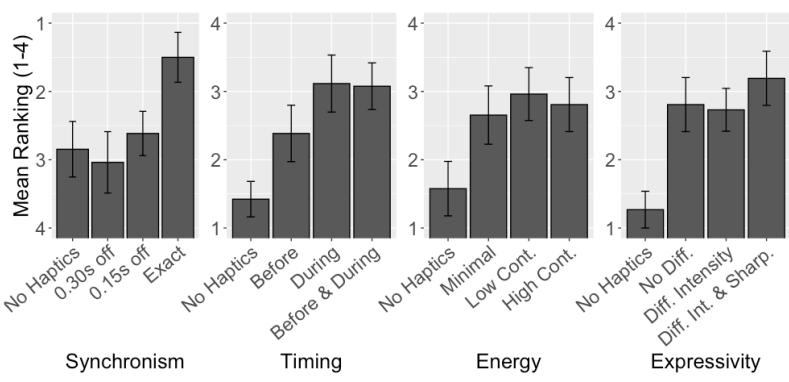

(b) Mean rankings for the four design principles that vary across haptic embellishment levels (i.e., haptic-only principles).

\begin{tabular}{||c||c|c|c|c|c|}
\hline & V2H2 & V2H1 & V2H0 & V1H2 & V1H1 \\
\hline \hline V2H1 & $0.026(*)$ & - & - & - & - \\
\hline V2H0 & $(* * *)$ & $(* * *)$ & - & - & - \\
\hline V1H2 & $(* * *)$ & $(* * *)$ & $(* * *)$ & - & - \\
\hline V1H1 & $0.014(*)$ & 0.821 & $(* * *)$ & $(* * *)$ & - \\
\hline V1H0 & $(* * *)$ & $(* * *)$ & 0.318 & $(* * *)$ & $(* * *)$ \\
\hline
\end{tabular}

(d) Adjusted p-values $(p<.05(*), p<.01(* *)$ and $p<.001(* * *))$ from multiple comparison tests of preferences across embellishment conditions from the data in Figure 5c.

Figure 5: User preference data for manifestation of the design principles in study 1. A ranking of one would indicate the highest preference. Larger bar heights mean higher preference - note that one is at the top of the y-axis. Error bars show the $\mathbf{9 5 \%}$ confidence interval.

cleared or if the paddle misses the ball. See Supplemental Materials for the source code of the game and haptic feedback.

Visual: Low Juicy versus High Juicy. The low juicy visuals are in black-and-white with no visual embellishments. There are no additional animation or particle effects beyond what is required for the game mechanics. The high juicy visuals are in colour, a basic visual juice effect $[27,32]$. It includes a particle effect when the ball spawns. When the ball collides with objects, it squishes and stretches and produces particle effects. On collision with the ball, the paddle wobbles back-and-forth. Object collisions also create a screen shake effect and an animation effect on all the bricks. When the ball hits a brick, the brick falls, shrinks, and fades out. See Figure 1 and the Video Figure for more details.

Haptic: Low Juicy versus High Juicy. The low juicy haptic feedback produces the same transient haptic effect ( $50 \%$ intensity, $50 \%$ sharpness) whenever the ball collides with any object. The design of the high juicy haptic feedback is informed by the results of study 1 and informal iterations. It includes staging and anticipation feedback that match the particle effects when the ball spawns. When the ball collides with any object, it produces a transient haptic effect that varies depending on the object it hit (e.g., brick, wall, or paddle) to create expressivity and realism. For example, collisions with the paddle produce softer (low frequency) vibrations to communicate its elasticity, while collisions with the wall produce sharp feedback (high frequency) to indicate its rigidity. The sharpness and intensity on collisions with the bricks is randomized to produce more dynamic feedback. When the ball hits the paddle, the haptic feedback oscillates (follow-through) in synchronization with the paddle's wobble animation. In general, the haptic feedback in the high juicy condition is more intense and ornate than the low juicy condition (exaggeration). See Figure 1 and the Video Figure.

Both juicy conditions exactly synchronize the visual and haptic effects. We do not implement the HE principles of slow in and out, experiment with timing, nor communicate energy of motion because of their mixed results in study 1 (see Figure $5 \mathrm{a}$ and Figure $5 b)$.

Study App. Similar to study 1, we created an iOS app that guides participants through the entire study - it collects consent, checks if vibrations are supported, presents the games, asks our questions (see below section on metrics), and collects data. See the Video Figure for a walkthrough of the interface.

\subsection{Metrics}

6.2.1 Player Experience. We measure PX using subscales from the Player Experience Inventory (PXI) [1]: appeal, immersion, mastery, and meaning. We did not include other constructs for two reasons. Firstly, many of the constructs such as autonomy, curiosity, and progress feedback are irrelevant to embellishments (both visual and haptic) because they do not modify the underlying game mechanics. Secondly, asking all ten constructs after each of the six games is a huge time commitment and possibly fatiguing for participants. 
6.2.2 Haptic Experience. We ask questions about haptic experience (HX) [34] using a questionnaire ${ }^{1}$, which consists of three questions for each of the HX model's experiential factors of autotelics, expressivity, harmony, immersion, and realism. See Supplemental Material for the exact questions.

6.2.3 Others. We measure enjoyability through a question: "I enjoyed playing the game". To verify that the participant is paying attention, we also include a trap question where participants must pick a specific response from the 7-point Likert scale (e.g., "for this question only, please select SLIGHTLY DISAGREE.”). The specific response required in the trap question is picked from a random number generator. We insert the enjoyability question and trap question into a random position within the set of questions we ask participants after a game.

\subsection{Participants}

We recruited 38 participants from the same sources and eligibility criteria as study 1 . Two participants were removed from the study for failing multiple trap questions and repeatedly selecting the same answer to every question. Of the remaining 36 participants, 24 selfidentified as women, 11 as men, 1 as non-binary. Participants were 18 to 44 years old with limited familiarity with haptics.

\subsection{Procedure}

The initial steps of this study are the same as study 1 to collect consent, demographics, and confirm that haptics are supported and enabled. Please refer to the first paragraph of study 1's procedure for more details.

Next, participants are shown all six versions of the Breakoutstyle game in randomized order. Each game must be played for a minimum of 90 seconds and is followed by a questionnaire. The minimum time requirement is enforced through the app - participants may only continue to the questionnaire once the time is up. The questionnaire presents our measures in the order of PXI, enjoyability, and HX (if the game included haptic feedback); within a specific measure (e.g., PXI or HX), questions are presented in randomized order. There is also a trap question in the questionnaire (same as in study 1) to check if the participant is paying attention and verify data quality. At the end of the study, participants are asked to optionally provide "any comments or feedback about the games, the haptics, or the study?"

\subsection{Results}

6.5.1 Data analysis procedure. Because the data did not satisfy the normality assumption for ANOVA, we used Friedman tests for significance testing, followed by a Cohen's D test for effect size. Pvalues were adjusted using the Benjamini-Hochberg [6] procedure to reduce the false discovery rate (FDR).

6.5.2 Game conditions. The six different games had significant $(p<0.05)$ effects on enjoyability, PXI appeal, PXI immersion, PXI meaning (see Table 1). There were no significant effects on PXI

\footnotetext{
${ }^{1}$ These questions are derived from an ongoing project conducting scale developmen for a proposed instrument for haptic experience (HX) [34]. While the project is not yet published at the time of this writing and should not be considered a validated scale at this point, we include these questions as our best guess for measuring relevant constructs for HX.
}

mastery of the game, which makes sense given that none of the conditions modified game mechanics or controls. Further analysis comparing only the four games with haptics shows significant effects of the game on HX expressivity and HX harmony.

6.5.3 Visual conditions. The two visual conditions (low juicy and high juicy) had no significant effects on any of our measurements. A deeper dive shows that although participants' enjoyabillity of low juicy visuals followed a unimodal distribution, their enjoyability of the high juicy visuals was bi-modal with a small hump at lower ratings and a large hump at higher ratings. These mixed results are supported by comments. Some participants preferred the juicy high visuals: "I liked seeing the paddle boing with the Haptic Touch and lost track of time while playing, but during all the non-colored games I was watching the timer to see how long I had left." Other participants found the low juicy visuals "the prettiest because it was simple" and "appreciated what the [high juicy visual] mode was going for, but the visuals sometimes felt too much."

6.5.4 Haptic conditions. The haptic conditions had significant effects on enjoyability, PXI appeal, PXI immersion, and PXI meaning (see Figure 6). Further analysis comparing only the low juicy and high juicy haptics shows no significant effects on any of the constructs, except for HX expressivity. This suggests that although the inclusion of haptic feedback enhanced enjoyability and several PXI constructs, the two different implementations of haptic feedback had little impact.

\section{DISCUSSION}

We discuss the findings from our two studies and some key takeaways. Based on some negative results on higher visual juice in study 2, we also discuss whether there is such a thing as too much juice. Finally, we acknowledge limitations in our work and recommend future research on juicy haptics.

\subsection{The design principles produced better vibrotactile embellishments}

We found that high haptic juice (versus low haptic juice) enhanced visual stimuli in four of the six visuo-haptic principles: anticipation, staging, follow-through, exaggeration (Figure 5a). The other two principles (realism and slow in and out) produced far more mixed results, which may be a result of participants struggling to distinguish the stimuli; data indicates that participants rated the stimuli within these two principles as the hardest to distinguish. On the four haptic-only principles (see Figure 5b), our key takeaways are that 1) haptic embellishments and visual embellishments should be synchronized exactly, 2) haptic embellishments should be used to communicate an object's energy levels (e.g., motion), and 3) vibrotactile embellishments should vary across both intensity and sharpness for different objects and events. Future research should further study and iterate on these design principles.

\subsection{Vibrotactile embellishments improved constructs of player experience.}

In short, we found that the inclusion of vibrotactile embellishments can enhance PX, but we did not find much significant difference between the low and high juicy haptic conditions on PX constructs. 


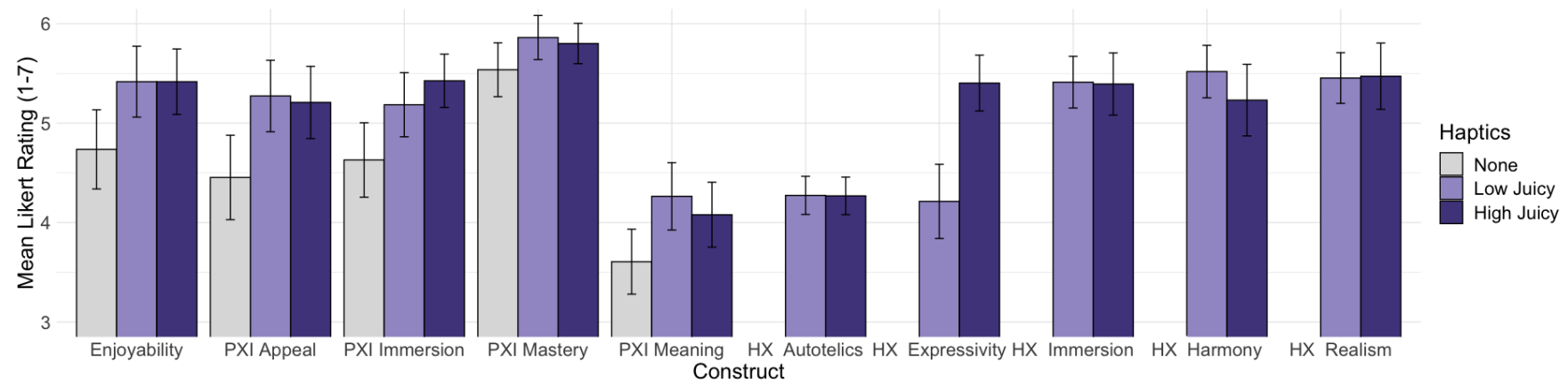

Figure 6: Study 2's mean ratings of haptic feedback on enjoyability, PXI, and HX constructs. Error bars show the 95\% confidence interval. We find significant differences between the "no haptics" condition and any of the juicy haptic conditions, but we do not observe difference between low and high juicy, except in HX expressivity.

Table 1: Quantitative data for all six Breakout-style games (split by visual and haptic juice levels). Note: to compute $p$-values for HX constructs, we excluded data from games with $\mathrm{HO}$ (no haptics).

\begin{tabular}{|c|c|c|c|c|c|c|c|c|c|}
\hline \multirow{2}{*}{$\begin{array}{l}\text { Visual Juice Levels } \\
\text { Haptic Juice Levels }\end{array}$} & \multicolumn{3}{|c|}{ V1 (low juicy visuals): mean(sd) } & \multicolumn{3}{|c|}{ V2 (high juicy visuals): mean(sd) } & \multicolumn{3}{|c|}{ Significance (adjusted p-values) } \\
\hline & Ho (none) & H1 (low) & H2 (high) & H0 (none) & H1 (low) & H2 (high) & Visuals & Haptics & Visuals $\mathrm{x}$ Haptics \\
\hline Enjoyability & $4.92(1.65)$ & $5.64(1.31)$ & $5.47(1.30)$ & $4.56(1.75)$ & $5.19(1.69)$ & $5.36(1.51)$ & 0.320 & $<0.001(* * *)$ & $0.004(* *)$ \\
\hline PXI Appeal & $4.74(1.71)$ & $5.56(0.99)$ & $5.28(1.27)$ & $4.17(1.87)$ & $4.99(1.90)$ & $5.14(1.80)$ & 0.317 & $<0.001(* * *)$ & $0.003(* *)$ \\
\hline PXI Immersion & $4.51(1.60)$ & $5.06(1.40)$ & $5.36(1.19)$ & $4.75(1.60)$ & $5.31(1.35)$ & $5.49(1.10)$ & 0.748 & $<0.001(* * *)$ & $<0.001(* * *)$ \\
\hline PXI Mastery & $5.78(0.85)$ & $5.81(0.79)$ & $5.93(0.88)$ & $5.30(1.36)$ & $5.91(1.08)$ & $5.68(0.85)$ & 0.337 & 0.219 & 0.096 \\
\hline PXI Meaning & $3.70(1.40)$ & $4.26(1.37)$ & $4.21(1.39)$ & $3.51(1.39)$ & $4.27(1.53)$ & $3.94(1.40)$ & 0.317 & $<0.001(* * *)$ & $<0.001(* * *)$ \\
\hline HX Autotelics & NA & $4.19(0.86)$ & $4.21(0.91)$ & NA & $4.36(0.77)$ & $4.32(0.69)$ & 0.147 & 1.000 & 0.1683 \\
\hline HX Expressivity & NA & $4.07(1.63)$ & $5.24(1.33)$ & NA & $4.35(1.56)$ & $5.56(1.04)$ & 0.558 & $<0.001(* * *)$ & $<0.001(* * *)$ \\
\hline HX Immersion & NA & $5.46(0.90)$ & $5.36(1.23)$ & NA & $5.36(1.29)$ & $5.56(1.45)$ & 0.558 & 0.999 & 0.820 \\
\hline HX Harmony & NA & $5.85(0.84)$ & $5.04(1.48)$ & NA & $5.19(1.28)$ & $5.43(1.58)$ & 0.558 & 0.999 & 0.09 \\
\hline HX Realism & NA & $5.65(0.97)$ & $5.20(1.49)$ & NA & $5.26(1.17)$ & $5.74(1.31)$ & 0.800 & 0.999 & 0.338 \\
\hline
\end{tabular}

Conditions with vibrotactile embellishments saw improvements in the PXI constructs of aesthetic appeal, immersion, meaning (see Figure 6 and Table 1). No statistically significant difference was found for PXI mastery, which was generally ranked very highly across all conditions, proving that our game was familiar and easy to play.

From the comments collected at the end of the study, we found that many participants specifically praised the haptic feedback:

"Didn't realize haptics could enrich the experience by
that much."
"The game feels good with the vibration and I like the
style of the game."
"I liked seeing the paddle bong with the Haptic Touch
and lost track of time while playing."
"The vibrations were really satisfying and felt good es-
pecially [V2H2]"

The high juicy haptic condition outperformed the low juicy haptic condition in HX expressivity, which shows that participants were able to discern the two conditions; however, there were no differences in any of the other constructs. This absence of significant results is surprising given the success of study 1's high haptic embellishment. One possible explanation is that the high juicy haptics in study 2 were designed to match the high juicy visuals, but because some participants found the high juicy visuals "too much" (see subsection 7.3), the high juicy haptics may have failed to live up to its potential. A more gloomy explanation is that the high juicy haptics could have been overly frequent and excessive. However, we do not believe this is the case because participant's comment criticizes the visual feedback for being too much, not the haptic feedback. Quantitative data supports that the high juicy haptic condition's enjoyability followed a unimodal distribution, suggesting that participants were not polarized (unlike the high juicy visual condition).

\subsection{Is there such a thing as too much juice?}

In study 2, we did not find any significant impact of juicy visuals on PX (see Table 1). In fact, we found that high juicy visuals were polarizing - some participants complained that the visuals were "too much", "over the top", and even "a bit dizzying". However, all complaints highlighted abundance, which is the guiding philosophy of juiciness. This raises the question: is there such a thing as too much juice? Kao et al. [32] found evidence that "Extreme" juice can downgrade PX (see section 3); one possible theory is that redundant audiovisual feedback can overburden working memory [30]. This 
topic requires more research; our recommendation is to continue making juicy games but to iterate and exercise judgment.

\subsection{Limitations and Future Work}

Limited to one type of game. The type of game and how haptic feedback fits in it will affect the added value on player experience (as Hicks et al. found for visual embellishments [21]). We focused on a Breakout-style game because it builds on prior work on juiciness [27]. It is also familiar and simple to readers, making it easier to appreciate the differences in juice conditions. That being said, it is important to verify these results across a diverse set of games.

Limited to implementing juice using embellishments. Juiciness includes other components than embellishments such as game state and game characteristics [33]. For this paper, we scoped down to one type of implementation - embellishments, which also had the downside of limiting the number of PX constructs that were relevant. Future work should investigate other aspects of juiciness and use other constructs of PX.

Future work: beyond vibrotactile feedback. We empirically studied vibrotactile feedback in iPhones but not other haptic modalities such as force-feedback or mid-air feedback. The upside is that vibrotactile actuators are widespread, making our work immediately applicable in today's consumer devices such as smartphones, smartwatches, and game controllers. Yet, vibrotactile feedback in phones are severely limited compared to other haptic modalities. For one, it only stimulates tactile perception, not kinesthetic perception a perception that deals with body movement and muscle feelings. Moreover, designers cannot control the precise location, temperature, or volumetric shape of feedback - some or all [31] of which are (proposed) capabilities in other haptic hardware. The design space in these other haptic modalities is far larger and richer, enabling designs that enhance player experience far beyond our results. We believe this an important and exciting area for future work.

Future work: haptic, audio, visual juice. We only studied visual and haptic juice to reduce the number of factors in our user study. However, it is well known $[11,34]$ that the harmony of haptic feedback and audio "can create very delightful and magical experiences" [40]. There is an immediate opportunity for research on juiciness or player experience that includes audio feedback (and even other sensory feedback such as taste and smell) along with haptic and visual feedback.

Future work: juiciness beyond games. Even though juiciness has primarily been studied in games literature, we did not limit our definition of juicy haptics to games because it could also be a useful concept for designing haptic feedback in other interactive media. Study 1 shows encouraging preliminary evidence that juicy haptics can enhance preference for short animation clips. Juicy haptics-the idea of excessive positive (sometimes redundant) haptic feedbackcould also bring benefits in gameful experiences (e.g., to promote engagement and motivation in a gamified fitness application), menu interfaces, and more.

\section{CONCLUSION}

We started by defining juicy haptics and haptic embellishments. We then assembled ten design principles for haptic embellishments and empirically evaluated their implementations in study 1 . Next, we used these design principles to design juicy haptics in a Breakoutstyle game for study 2 and found that haptic feedback can improve player experience, in particular the constructs of enjoyability, aesthetic appeal, immersion, meaning, and enjoyability. We hope to bring haptics to the forefront of the research on juiciness and player experience.

\section{ACKNOWLEDGMENTS}

We thank the University of Waterloo HCI community, especially the Haptic Computing Lab and Games Institute, for their continued guidance and feedback. We acknowledge the support from Canada Foundation for Innovation (CFI), John R. Evan's Leaders Fund, and the Natural Sciences and Engineering Research Council of Canada (NSERC) Discovery Grant program.

\section{REFERENCES}

[1] Vero Vanden Abeele, Katta Spiel, Lennart Nacke, Daniel Johnson, and Kathrin Gerling. 2020. Development and validation of the player experience inventory: A scale to measure player experiences at the level of functional and psychosocial consequences. International Journal of Human-Computer Studies 135 (2020), 102370. https://doi.org/10.1016/j.ijhcs.2019.102370

[2] Sheldon Andrews, Javier Mora, Jochen Lang, and WonSook Lee. 2006. HaptiCast: A physically-based 3D game with haptic feedback. In Proceedings of FuturePlay 2006 (FuturePlay 2006). FuturePlay, London, ON, Canada.

[3] Simeon Atanasov. 2013. Juiciness: Exploring and designing around experience of feedback in video games. Master's thesis. Malmö högskola/Kultur och samhälle.

[4] Mahdi Azmandian, Mark Hancock, Hrvoje Benko, Eyal Ofek, and Andrew D. Wilson. 2016. Haptic Retargeting: Dynamic Repurposing of Passive Haptics for Enhanced Virtual Reality Experiences. In Proceedings of the 2016 CHI Conference on Human Factors in Computing Systems (San Jose, California, USA) (CHI '16). Association for Computing Machinery, New York, NY, USA, 1968-1979. https: //doi.org/10.1145/2858036.2858226

[5] Scott Bateman, Regan L. Mandryk, Carl Gutwin, Aaron Genest, David McDine, and Christopher Brooks. 2010. Useful Junk? The Effects of Visual Embellishment on Comprehension and Memorability of Charts. In Proceedings of the SIGCHI Conference on Human Factors in Computing Systems (Atlanta, Georgia, USA) (CHI '10). Association for Computing Machinery, New York, NY, USA, 2573-2582. https://doi.org/10.1145/1753326.1753716

[6] Yoav Benjamini and Yosef Hochberg. 1995. Controlling the False Discovery Rate: A Practical and Powerful Approach to Multiple Testing. Fournal of the Royal Statistical Society. Series B (Methodological) 57, 1 (1995), 289-300. http: //www.jstor.org/stable/2346101

[7] Christopher C Berger, Mar Gonzalez-Franco, Eyal Ofek, and Ken Hinckley. 2018. The uncanny valley of haptics. Science Robotics 3, 17 (2018), Art--No. https: //doi.org/10.1126/scirobotics.aar7010

[8] Stephen Brewster and Lorna M. Brown. 2004. Tactons: Structured Tactile Messages for Non-Visual Information Display. In Proceedings of the Fifth Conference on Australasian User Interface - Volume 28 (Dunedin, New Zealand) (AUIC '04). Australian Computer Society, Inc., Darlinghurst, Australia, 15-23.

[9] L. M. Brown, S. A. Brewster, and H. C. Purchase. 2005. A first investigation into the effectiveness of Tactons. In First foint Eurohaptics Conference and Symposium on Haptic Interfaces for Virtual Environment and Teleoperator Systems. World Haptics Conference (Pisa, Italy). IEEE, Los Alamitos, CA, USA, 167-176. https: //doi.org/10.1109/WHC.2005.6

[10] N. Caporusso, L. Mkrtchyan, and L. Badia. 2010. A Multimodal Interface Device for Online Board Games Designed for Sight-Impaired People. IEEE Transactions on Information Technology in Biomedicine 14, 2 (2010), 248-254.

[11] Angela Chang and Conor O'Sullivan. 2005. Audio-Haptic Feedback in Mobile Phones. In CHI '05 Extended Abstracts on Human Factors in Computing Systems (Portland, OR, USA) (CHI EA '05). Association for Computing Machinery, New York, NY, USA, 1264-1267. https://doi.org/10.1145/1056808.1056892

[12] H. A. David. 1971. Ranking the Players in a Round Robin Tournament. Revue de l'Institut International de Statistique / Review of the International Statistical Institute 39, 2 (1971), 137-147. https://doi.org/10.2307/1402170

[13] Sebastian Deterding. 2015. The lens of intrinsic skill atoms: A method for gameful design. Human-Computer Interaction 30, 3-4 (2015), 294-335. 
[14] Mohamad Eid, Ahmad El Issawi, and Abdulmotaleb El Saddik. 2014. Slingshot 3D: A synchronous haptic-audio-video game. Multimedia tools and applications 71, 3 (2014), 1635-1649.

[15] Mohamad A Eid, Senior Member, and Hussein A L Osman. 2016. Affective Haptics : Current Research and Future Directions. IEEE Access 4 (2016), 26-40. https://doi.org/10.1109/ACCESS.2015.2497316

[16] Rob Eisinga, Tom Heskes, Ben Pelzer, and Manfred Te Grotenhuis. 2017. Exact p-values for pairwise comparison of Friedman rank sums, with application to comparing classifiers. BMC bioinformatics 18, 1 (2017), 68. https://doi.org/10. 1186/s12859-017-1486-2

[17] Kyle Gray, Kyle Gabler, Shalin Shodhan, and Matt Kucic. 2005. How to prototype a game in under 7 days. https://www.gamasutra.com/view/feature/130848/how to_prototype_a_game_in_under_7_.php

[18] Philippe Guillotel, Fabien Danieau, Julien Fleureau, and Ines Rouxel. 2016. Introducing Basic Principles of Haptic Cinematography and Editing. In Proceedings of the Eurographics Workshop on Intelligent Cinematography and Editing (Lisbon, Portugal) (WICED '16). Eurographics Association, Goslar, Germany, 15-21. https://doi.org/10.2312/wiced.20161096

[19] Ulf Hagen. 2011. Designing for player experience: How professional game developers communicate design visions. fournal of Gaming \& Virtual Worlds 3, 3 (2011), 259-275.

[20] Marc Hassenzahl, Michael Burmester, and Franz Koller. 2003. AttrakDiff: Ein Fragebogen zur Messung wahrgenommener hedonischer und pragmatischer Qualität Vieweg+Teubner Verlag, Wiesbaden, 187-196. https://doi.org/10.1007/978-3322-80058-9_19

[21] Kieran Hicks, Kathrin Gerling, Patrick Dickinson, and Vero Vanden Abeele 2019. Juicy Game Design: Understanding the Impact of Visual Embellishments on Player Experience. In Proceedings of the Annual Symposium on Computer Human Interaction in Play (Barcelona, Spain) (CHI PLAY '19). Association for Computing Machinery, New York, NY, USA, 185-197. https://doi.org/10.1145/ 3311350.3347171

[22] Kieran Hicks, Kathrin Gerling, Graham Richardson, Tom Pike, Oliver Burman, and Patrick Dickinson. 2019. Understanding the effects of gamification and juiciness on players. In 2019 IEEE Conference on Games (CoG). IEEE, IEEE, London, UK, 1-8. https://doi.org/10.1109/CIG.2019.8848105

[23] Ali Israr, Seung-Chan Kim, Jan Stec, and Ivan Poupyrev. 2012. Surround Haptics: Tactile Feedback for Immersive Gaming Experiences. In CHI '12 Extended Abstracts on Human Factors in Computing Systems (Austin, Texas, USA) (CHI EA '12). Association for Computing Machinery, New York, NY, USA, 1087-1090. https://doi.org/10.1145/2212776.2212392

[24] Ali Israr, Siyan Zhao, Kyna McIntosh, JaeKyun Kang, Zachary Schwemler, Eric Brockmeyer, Mark Baskinger, and Moshe Mahler. 2015. Po2: Augmented Haptics for Interactive Gameplay. In ACM SIGGRAPH 2015 Emerging Technologies (Los Angeles, California) (SIGGRAPH '15). Association for Computing Machinery, New York, NY, USA, Article 21, 1 pages. https://doi.org/10.1145/2782782.2792489

[25] Ali Israr, Siyan Zhao, Kaitlyn Schwalje, Roberta Klatzky, and Jill Lehman. 2014 Feel Effects: Enriching Storytelling with Haptic Feedback. ACM Trans. Appl. Percept. 11, 3, Article 11 (Sept. 2014), 17 pages. https://doi.org/10.1145/2641570

[26] Ollie Johnston and Frank Thomas. 1981. The illusion of life: Disney animation. Walt Disney Editions, New York, USA. 47-69 pages.

[27] Martin Jonasson and Petri Purho. 2012. Juice it or lose it. https://youtu.be/ Fy0aCDmgnxg

[28] Jesper Juul. 2010. A casual revolution: Reinventing video games and their players. MIT press, Cambridge, MA.

[29] Jesper Juul and Jason Scott Begy. 2016. Good Feedback for bad Players? A preliminary Study of 'juicy' Interface feedback. In Proceedings of first joint FDG/DiGRA Conference. Digital Games Research Association DiGRA, Dundee, Scotland, UK.

[30] Slava Kalyuga, Paul Chandler, and John Sweller. 1999. Managing split-attention and redundancy in multimedia instruction. Applied Cognitive Psychology: The Official fournal of the Society for Applied Research in Memory and Cognition 13, 4 (1999), 351-371

[31] Takaaki Kamigaki, Shun Suzuki, and Hiroyuki Shinoda. 2020. Noncontact Thermal and Vibrotactile Display Using Focused Airborne Ultrasound. In Haptics Science, Technology, Applications, Ilana Nisky, Jess Hartcher-O’Brien, Michaël Wiertlewski, and Jeroen Smeets (Eds.). Springer International Publishing, Cham, 271-278.

[32] Dominic Kao. 2020. The effects of juiciness in an action RPG. Entertainment Computing 34 (2020), 100359. https://doi.org/10.1016/j.entcom.2020.100359

[33] Hicks Kieran, Dickinson Patrick, Holopainen Jussi, and Gerling Kathrin. 2018 Good Game Feel: An Empirically Grounded Framework for Juicy Design In DiGRA \&\#3918 - Proceedings of the 2018 DiGRA International Conference: The Game is the Message. Digital Games Research Association DiGRA, Turin Italy. http://www.digra.org/wp-content/uploads/digital-library/DIGRA_2018_ Paper_35.pdf

[34] Erin Kim and Oliver Schneider. 2020. Defining Haptic Experience: Foundations for Understanding, Communicating, and Evaluating HX. In Proceedings of the 2020 CHI Conference on Human Factors in Computing Systems (Honolulu, HI USA) (CHI '20). Association for Computing Machinery, New York, NY, USA, 1-13. https://doi.org/10.1145/3313831.3376280

[35] Ben Kuchera. 2017. How the Nintendo Switch's HD rumble makes Tumbleseed feel real. https://www.polygon.com/2017/5/1/15499328/tumbleseed-hd-rumblenintendo-switch

[36] Karon Maclean and Mario Enriquez. 2003. Perceptual design of haptic icons. In In Proceedings of Eurohaptics. EuroHaptics, Dublin, Ireland, 351-363. https: //eurohaptics.org/meeting2003/

[37] Karon E. MacLean, Oliver S. Schneider, and Hasti Seifi. 2017. Multisensory Haptic Interactions: Understanding the Sense and Designing for It. Vol. 1. Association for Computing Machinery and Morgan \& Claypool, New York, NY, USA, 97-142. https://doi.org/10.1145/3015783.3015788

[38] Emanuela Maggioni, Erika Agostinelli, and Marianna Obrist. 2017. Measuring the added value of haptic feedback. In 2017 Ninth International Conference on Quality of Multimedia Experience (QoMEX). IEEE, IEEE, Erfurt, Germany, 1-6. https://doi.org/10.1109/OoMEX.2017.7965670

[39] Tony Morelli, John Foley, Luis Columna, Lauren Lieberman, and Eelke Folmer. 2010. VI-Tennis: A Vibrotactile/Audio Exergame for Players Who Are Visually Impaired. In Proceedings of the Fifth International Conference on the Foundations of Digital Games (Monterey, California) (FDG '10). Association for Computing Machinery, New York, NY, USA, 147-154. https://doi.org/10.1145/1822348.1822368

[40] Camille Mousette and Hugo Verweij. 2019. Designing Audio-Haptic Experiences. https://developer.apple.com/videos/play/wwdc2019/810/

[41] Marianna Obrist, Sue Ann Seah, and Sriram Subramanian. 2013. Talking about Tactile Experiences. In Proceedings of the SIGCHI Conference on Human Factors in Computing Systems (Paris, France) (CHI '13). Association for Computing Machinery, New York, NY, USA, 1659-1668. https://doi.org/10.1145/2470654.2466220

[42] Wanjoo Park, Laehyun Kim, Hyunchul Cho, and Sehyung Park. 2009. Design of haptic interface for brickout game. In 2009 IEEE International Workshop on Haptic Audio visual Environments and Games. IEEE, Lecco, Italy, 64-68. https: //doi.org/10.1109/HAVE.2009.5356137

[43] Horea Pauna, Pierre-majorique Léger, Sylvain Sénécal, Marc Fredette, François Courtemanche, Shang-Lin Chen, Élise Labonté-Lemoyne, and Jean-François Ménard. 2018. The psychophysiological effect of a vibro-kinetic movie experience: the case of the D-BOX movie seat. In Information Systems and Neuroscience. Springer, Cham, Switzerland, 1-7. https://doi.org/10.1007/978-3-319-67431-5_1

[44] Jussi Rantala, Katri Salminen, Roope Raisamo, and Veikko Surakka. 2013. Touch gestures in communicating emotional intention via vibrotactile stimulation. International fournal of Human-Computer Studies 71, 6 (June 2013), 679-690. https://doi.org/10.1016/j.ijhcs.2013.02.004

[45] Oliver Schneider, Jotaro Shigeyama, Robert Kovacs, Thijs Jan Roumen, Sebastian Marwecki, Nico Boeckhoff, Daniel Amadeus Gloeckner, Jonas Bounama, and Patrick Baudisch. 2018. DualPanto: A Haptic Device That Enables Blind Users to Continuously Interact with Virtual Worlds. In Proceedings of the 31st Annual ACM Symposium on User Interface Software and Technology (Berlin, Germany) (UIST '18). Association for Computing Machinery, New York, NY, USA, 877-887. https://doi.org/10.1145/3242587.3242604

[46] H. Seifi, M. Chun, C. Gallacher, O. Schneider, and K. E. MacLean. 2020. How Do Novice Hapticians Design? A Case Study in Creating Haptic Learning Environments. IEEE Transactions on Haptics 13, 4 (2020), 791-805. https: //doi.org/10.1109/TOH.2020.2968903

[47] Hasti Seifi and Karon E MacLean. 2017. Exploiting haptic facets: Users' sensemaking schemas as a path to design and personalization of experience. International fournal of Human-Computer Studies 107 (2017), 38-61. https: //doi.org/10.1016/j.ijhcs.2017.04.003

[48] Steve Swink. 2008. Game feel: a game designer's guide to virtual sensation. CRC Press, Boca Raton, FL, USA.

[49] u/hiperlalol. 2018. r/iosgaming - What are your thoughts Haptic feedback on games? https://www.reddit.com/r/iosgaming/comments/7o6o5f/what_are_ your_thoughts_haptic_feedback_on_games

[50] Yongjae Yoo, Taekbeom Yoo, Jihyun Kong, and Seungmoon Choi. 2015. Emotional responses of tactile icons: Effects of amplitude, frequency, duration, and envelope. In 2015 IEEE World Haptics Conference (WHC). IEEE, Evanston, IL, USA, 235-240. https://doi.org/10.1109/WHC.2015.7177719 